\title{
Aberrant cystatin-C expression in blood from patients with breast cancer is a suitable marker for monitoring tumor burden
}

\author{
WOO SUN KWON ${ }^{1}$, TAE SOO KIM ${ }^{1}$, CHUNG HYUN NAHM ${ }^{2}$, YEONSOOK MOON ${ }^{2}$ and JIN JU KIM ${ }^{2}$ \\ ${ }^{1}$ Song-Dang Institute for Cancer Research, Cancer Metastasis Research Center, Yonsei University College of Medicine, \\ Seoul 03722; ${ }^{2}$ Department of Laboratory Medicine, Inha University College of Medicine, Incheon 22332, Republic of Korea
}

Received March 5, 2016; Accepted March 30, 2017

DOI: $10.3892 / \mathrm{ol} .2018 .9380$

\begin{abstract}
The present study was performed to evaluate the efficacy of circulating cystatin- $\mathrm{C}$ as a tumor monitoring biomarker at different clinical time points in patients with breast cancer over a long-term follow-up period. In addition, the secretory rate of circulating cystatin- $\mathrm{C}$ from cancer tissue was investigated by comparing the blood and tissue expression levels of cystatin-C. Blood samples from healthy volunteers (40 males and 40 females) were obtained at yearly health examinations if laboratory and imaging abnormalities were not detected. Blood samples from 34 patients with breast cancer were obtained at 205 different time points of clinical progression. Blood levels of cystatin- $\mathrm{C}$ were measured using ELISA and the tissue levels were measured using immunohistochemistry. No age-associated effect was observed in male and female blood cystatin-C levels. The positivity rate was $46 \%$ in patients (38/83) and $40 \%$ in samples collected at different time points $(82 / 205)$. Blood cystatin-C levels were lowest following surgery compared with patients with systemic metastasis $(\mathrm{P}<0.001)$. The sensitivity, specificity and accuracy rates of ELISA were 53.6, 63.6 and $53.9 \%$, respectively. The concordance rate between blood and tissue expression was $38 \%$. The main reason for discordance between tissue and serum expression of cytostatin- $\mathrm{C}$ came from low serum positivity in samples showing tissue cytostatin-C $(3 / 11,27 \%)$. The specificity between cytostatin- $\mathrm{C}$ and CA-125 was highest in tumor absence state. In conclusion, elevated blood levels of cystatin-C were observed in $40 \%$ of breast cancer cases and were tumor-volume dependent. However, the concordance rate between tissue and blood was quite low, suggesting tumor heterogeneity of cystatin-C expression or co-acting pathway activation, such as cathepsin D. As one-third of breast cancer tissues express cystatin- $\mathrm{C}$ without cancer antigen 15-3 elevation, cystatin- $\mathrm{C}$ may represent a good tumor-monitoring marker in breast cancer.
\end{abstract}

Correspondence to: Professor Jin Ju Kim, Department of Laboratory Medicine, Inha University College of Medicine, 27 Inhang-ro, Jung-ku, Incheon 22332, Republic of Korea

E-mail: jkimmd@inha.ac.kr

Key words: cystatin-C, breast cancer, tumor monitoring, blood

\section{Introduction}

Cystatins are a superfamily of cysteine proteinase inhibitors found in animals and plants. Cystatin-C, a secreted molecule of the cystatin family, is composed of 120 amino acids and has a molecular weight of 13,260 Da. Cystatin-C is increased in patients with cancer and is suggested to be a better renal functional marker than creatinine. In pediatric patients with cancer, cystatin- $\mathrm{C}$ is a suitable marker for monitoring renal function during chemotherapy (1). The importance of the accurate assessment of renal function in patients with cancer is increasing, and the prediction of the glomerular filtration rate with combined evaluation of cystatin- $\mathrm{C}$ and creatinine is being tested for anticancer agents, such as cisplatin (2). By contrast, a low level of cystatin-C is associated with atherosclerosis and aortic aneurysm. Additionally, it has been suggested that cystatin-C adversely affects metabolic factors, in particular abdominal obesity, and thus contributes to the development of metabolic syndrome (3).

Cathepsin B is a lysosomal cysteine protease in normal tissues, and an important matrix protease in cancers, including lung cancer, colon cancer and hepatocellular carcinoma (4). Activated cathepsin B is considered to be involved in cancer invasion and the destruction of the basement membrane, by degrading collagen, laminin and proteoglycans. Cathepsin B is regulated by cysteine protease inhibitors, such as stefin A, stefin B and cystatin-C. In colorectal cancer, cathepsin B expression may contribute to early stage invasion and systemic metastasis, without the effect of cystatin-C (5). It has been reported that non-malignant colorectal tissues do not express cystatin- $\mathrm{C}$, whilst cancer tissues expressed high levels of both cystatin- $\mathrm{C}$ and cysteine proteinase inhibitors (6). In a previous study of esophageal cancer, the intensity of cystatin-C immunostaining in cancer tissue was increased compared with the adjacent normal esophageal tissues. In addition, the mRNA expression levels of cystatin-C were increased in cancerous tissue compared with normal tissue (7). In prostate cancer, a significant reduction in the immunohistochemical expression of cystatin- $\mathrm{C}$ in non-neuroendocrine prostate cancer has been reported (8). However, there was strong expression of cystatin-C in neuroendocrine-like cells compared with benign prostate tissues, suggesting a strong connection between cystatin-C expression and neuroendocrine differentiation (8). In breast cancer, an imbalance between cathepsin B and cystatin-C 
expression has been reported in the early stages of cancer progression. However, no correlation between cathepsin B and cystatin-C expression levels in histological classification, tumor size, lymph node metastasis and hormonal receptor status was observed (9). Generally, an increase in the ratio of cathepsins to cystatins contributes to tumor invasiveness and metastasis (10). However, data are conflicting regarding the role of cystatins in cancer invasion and metastasis. High levels of cystatin-B and -C predict poor survival of patients with colorectal cancer, and increased cystatin-F showed a strong correlation with liver metastasis in colorectal cancer (11). Additionally, high levels of cystatin-A exhibited a strong correlation with tumor size and increased mitotic activity in breast cancer (12).

Extracellular levels of stefins and cystatin-C are also associated with cancer progression. In hepatocellular carcinoma, increased serum stefin A levels correlated with tumor size and number (13), and increased serum cystatin-C levels correlated with tumor stage in melanoma (14). In colorectal cancer, a correlation between high levels of extracellular cystatin- $\mathrm{C}$ and poor survival was observed (15). In elderly patients with lung cancer, serum cystatin- $C$ levels increased in an age-dependent manner between 65 and $\geq 75$ years (16). In serum samples from patients with breast cancer, high cystatin-C levels were found in larger sized tumors, in older patients and in post-menopausal women. Significantly lower levels of cathepsin X and H were found in patients with inflammatory breast cancer, and a trend was also observed for cathepsin B and cystatin-C (17).

The present study aimed to evaluate circulating cystatin- $\mathrm{C}$ as a biomarker at different clinical time points in patients with breast cancer over a long-term follow-up period. In addition, the excretory rate of circulating cystatin- $\mathrm{C}$ was investigated by comparing the blood and tissue expression levels of cystatin-C. To the best of our knowledge, the present study is the first to simultaneously assess the expression levels of cystatin-C in tissue and serum samples from patients with breast cancer at different time points of clinical progress.

\section{Materials and methods}

Collection of blood and tissues. Blood samples from healthy volunteers (40 males and 40 females) were obtained at Inha University Hospital (Incheon, Korea), subsequent to obtaining approval if no laboratory and imaging abnormalities were observed following check-up in 2003. Blood samples from 34 female patients with breast cancer were obtained at 205 different time points of initial diagnosis state, during chemotherapy period and off-chemotherapy state between January 1999 and January 2003, and stored at $-80^{\circ} \mathrm{C}$. Heparinized vacuum tubes and needles (BD Biosciences, Franklin Lakes, NJ, USA) were used to avoid platelet damage and venous occlusion, as in clinical sample setting. Following surgical excision (29 specimens) or biopsy (23 specimens) between January 1992 and December 2002, specimens were fixed in $10 \%$ formalin and embedded in paraffin. Approval for the present study was obtained from the Institutional Review Board of Severance Hospital (Seoul, Korea) and Inha University Hospital (IRB 4-2009-0256 and 10-617, respectively) and samples were used subsequent to obtaining consent from volunteers and patients.
Determination of the normal range of serum cystatin- $C$. Using 40 healthy male and 40 healthy female volunteer blood samples, the normal cut-off point of cystatin-C was determined. The cut-off point was determined using the mean \pm 2 standard deviations. The serum cystatin-C level was determined as positive when the blood value of the patient was higher than the cut-off value (18).

Immunohistochemistry. Sections $(4-\mu \mathrm{m})$ were stained by the horseradish peroxidase (HRP) labeling method. The specimens were deparaffinized and immersed in a solution of $0.1 \%$ hydrogen peroxide in methanol to eliminate endogenous peroxidase activity. The antibody used was rabbit anti-human cystatin-C polyclonal antibody (cat. no. ab68290; Abcam, Cambridge, MA, USA) with a 1:2,000 dilution. In total, a maximum of 500 cancer cells were counted. Positive staining was defined as the presence of cytoplasmic immunoreactivity in a minimum of $10 \%$ of cancer cells.

ELISA assay. A sandwich ELISA was used to measure cystatin-C, cancer antigen (CA) 15-3 and CA125 levels, according to the manufacturer's instructions. In brief, murine polyclonal antibody specific for cystatin-C (RD 191009100; dilution, 1:400; BioVendor, Brno, Czech Republic), and monoclonal antibodies specific for CA15-3 (cat. no. IS-F3329; LSBio, Seattle, WA, USA) and CA125 (cat. no. CA239T; Calbiotech, Inc., El Cajon, CA, USA) were used to coat the 96-well microplates. Each standard and sample was added to the plate and incubated for $1 \mathrm{~h}$ at room temperature. Following washing to remove unbound proteins, enzyme-linked antibodies specific for cystatin-C, CA15-3 and CA125 were added to the wells. Absorbance was measured at $450 \mathrm{~nm}$ and the detection limit of cystatin-C was $0.25 \mathrm{ng} / \mathrm{ml}$. A standard curve was constructed by plotting absorbance values vs. the cystatin-C, CA15-3 and CA125 concentrations of the standards, and the concentrations of the test samples were determined using this standard curve. All samples were run in triplicate. The intra- and inter-assay variations of cystatin-C were 3.4 and $6.9 \%$, respectively. Dilutional linearity was $98 \%$. The upper normal range were $25 \mathrm{U} / \mathrm{ml}$ for CA15-3 and $35 \mathrm{IU} / \mathrm{ml}$ for CA125.

Statistical analysis. The statistical analysis and graphing were performed using SPSS software, version 20 (IBM Corp., Armonk, NY, USA) and GraphPad Prism version 5 (GraphPad Software, Inc., La Jolla, CA, USA). The differences between the groups were analyzed using one-way analysis of variance (Wilcoxon rank-sum test and paired t-test) and the post-hoc test was performed by Turkey test. Correlation between variables was estimated using the Spearman's rank correlation coefficient. $\mathrm{P}<0.05$ was considered to indicate a statistically significant difference.

\section{Results}

Volunteers and patients with breast cancer. The median age of the healthy male and female volunteers was 41 (range, 25-66 years) and 35 years (range, 21-56 years), respectively. A total of 205 samples were obtained from 34 patients with breast cancer (median age, 45 years; range, 32-65 years) in 12 different disease states during long-term follow-up and 


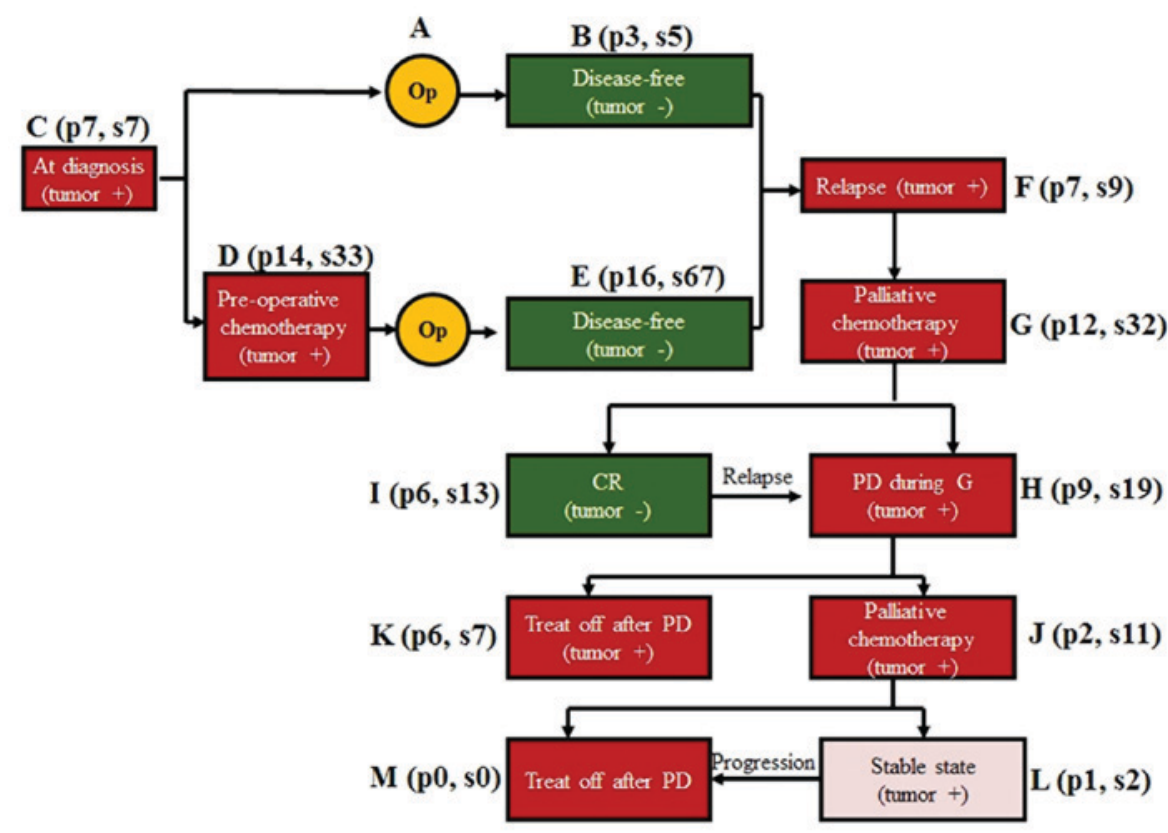

Figure 1. The 12 different time points where blood samples were taken from 34 breast cancer patients. Op, operation; PD, progressive disease; CR, complete remission; $\mathrm{p}$, patient number; s, sample number; treat off, no chemotherapy.

treatment (Fig. 1). Blood was also obtained prior to surgery in 7 patients with prostate cancer and 13 patients with colon cancer.

Normal range of cystatin- $C$. The mean levels of cystatin-C in healthy female and male volunteers were $0.61 \pm 0.09$ and $0.73 \pm 0.09 \mathrm{mg} / 1$, respectively. The levels were increased in men compared with women $(\mathrm{P}<0.001)$ and in patients with breast cancer compared with female volunteers $(\mathrm{P}=0.034)$ (Fig. 2). Using a cut-off point of the mean \pm 2 standard deviations, the cut-off point was $0.91 \mathrm{mg} / 1$ in men and $0.79 \mathrm{mg} / 1$ in women. There was no evidence of an effect of age in men or women (Table I). The positivity rate was $46 \%$ in patients (38/83) and $40 \%$ in samples collected from the different time points (82/205; Table II). Blood cystatin-C levels were lowest following surgery compared with patients with systemic metastasis $(\mathrm{P}<0.001$; Fig. 3). The sensitivity, specificity and accuracy rate of the ELISA was 53.6, 63.6 and 53.9\%, respectively (Table III). Among the 7 patients with prostate cancer, only 1 patient (14.2\%) showed cystatin-C positivity by ELISA. In the 13 patients with resectable colon cancer, no patients exhibited cystatin-C positivity in the blood.

Detection of cystatin-C by immunohistochemistry in patients with breast cancer. Immunohistochemical analysis was conducted at 3 different clinical time points (29 specimens at diagnosis, 21 specimens following pre-operative chemotherapy and 2 specimens at relapse), including 2 patients with bilateral breast cancer. The positivity rate was $41.4 \%(12 / 29)$ at diagnosis, $66.7 \%$ (14/21) following pre-operative chemotherapy, and $100 \%$ (2/2) following relapse. In 6 patients it was possible to compare cystatin-C expression between pre- and post-chemotherapy samples. The concordance rate was $66.7 \%$ (Table IV). In the 2 patients who relapsed, 1 patient showed cystatin- $\mathrm{C}$ expression in

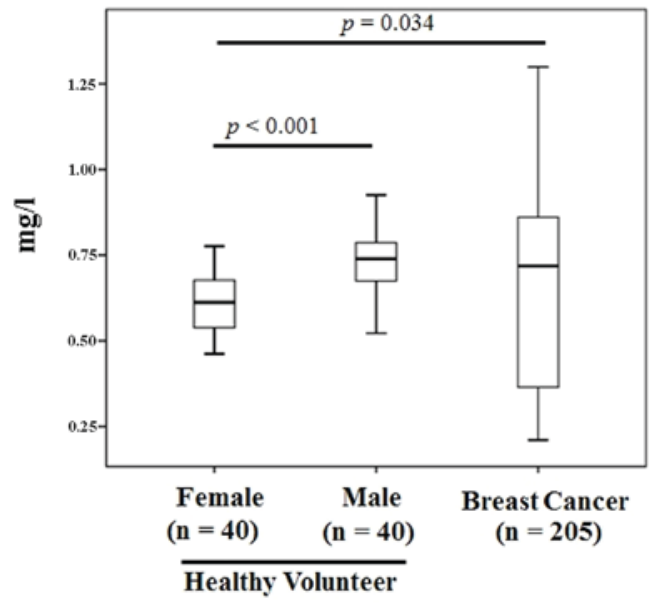

Figure 2. Comparison of cystatin-C levels among healthy volunteers and cancer patients.

both the primary and relapsed tumors. The other patient showed no expression in the pre- or post-chemotherapy samples, while cystatin- $\mathrm{C}$ expression was detected in the relapsed tumor. In the 2 patients with bilateral breast cancer, 1 patient exhibited simultaneous cystatin- $\mathrm{C}$ expression from both breast tumors, while the second showed discordance in cystatin- $\mathrm{C}$ expression in both breast cancers.

Comparison of paired-tissue immunohistochemistry and blood ELISA positivity. In 16 patients, tissue immunohistochemistry and blood ELISA were compared, resulting in a concordance rate of $38 \%$. The main reason of discordance between tissue and serum expression of cytostatin- $\mathrm{C}$ came from low serum positivity in samples showing tissue cytostatin- $\mathrm{C}$ positivity $(3 / 11,27 \%)$. Additionally, the specificity rate was $60 \%$. This indicates that in the early stages of breast cancer, the secretion 
Table I. Comparison of blood cystatin-C levels by ages in healthy volunteers.

\begin{tabular}{|c|c|c|c|c|c|c|}
\hline \multirow[b]{2}{*}{ Sex } & \multicolumn{6}{|c|}{ Age, years } \\
\hline & $21-30$ & $31-40$ & $41-50$ & $51-60$ & $61-70$ & Total \\
\hline \multicolumn{7}{|l|}{ Male } \\
\hline Number, $\mathrm{n}$ & 10 & 10 & 13 & 5 & 2 & 40 \\
\hline Mean \pm SD & $0.67 \pm 0.10$ & $0.76 \pm 0.07$ & $0.78 \pm 0.09$ & $0.71 \pm 0.04$ & $0.66 \pm 0.02$ & $0.73 \pm 0.09$ \\
\hline Median & 0.68 & 0.77 & 0.77 & 0.71 & 0.66 & 0.74 \\
\hline \multicolumn{7}{|l|}{ Female } \\
\hline Number, $\mathrm{n}$ & 12 & 16 & 10 & 2 & 0 & 40 \\
\hline Mean \pm SD & $0.60 \pm 0.09$ & $0.65 \pm 0.09$ & $0.55 \pm 0.07$ & $0.67 \pm 0.03$ & - & $0.61 \pm 0.09$ \\
\hline Median & 0.61 & 0.65 & 0.55 & 0.67 & - & 0.61 \\
\hline
\end{tabular}

Table II. Comparison of blood cystatin-C positivity at 12 different clinical time points

\begin{tabular}{llccc}
\hline & Number of & $\begin{array}{c}\text { Cystatin-C-positive } \\
\text { patients, } \\
\text { pumor state }\end{array}$ & Patient group & $\begin{array}{c}\text { Cystatin-C-positive } \\
\text { samples, } \mathrm{n}(\%) \\
\text { positive/total }\end{array}$ \\
\hline Presence of tumor & & 25 & & \\
At diagnosis & Group C & 7 & $3 / 7(43)$ & $3 / 7(43)$ \\
During pre-operative chemotherapy & Group D & 8 & $8 / 14(57)$ & $14 / 33(42)$ \\
At first relapse & Group F & 3 & $1 / 7(14)$ & $1 / 9(11)$ \\
During 1st line palliative chemotherapy & Group G & 5 & $5 / 12(42)$ & $16 / 32(50)$ \\
At PD after 1st line chemotherapy & Group H & 1 & $6 / 9(67)$ & $10 / 19(53)$ \\
Treat-off after 1st line chemotherapy & Group K & 1 & $2 / 6(33)$ & $2 / 7(29)$ \\
During 2nd line palliative chemotherapy & Group J & 0 & $2 / 2(100)$ & $11 / 11(100)$ \\
SD after 2nd line chemotherapy & Group L & 0 & $0 / 1(0)$ & $0 / 2(0)$ \\
PD after 2nd line palliative chemotherapy & Group M & 0 & $0 / 0(0)$ & $0 / 0(0)$ \\
Absence of tumor & & 9 & & $0 / 5(0)$ \\
After initial surgery & Group B & 3 & $0 / 3(0)$ & $15 / 67(22)$ \\
After surgery and neoadjuvant chemotherapy & Group E & 4 & $6 / 16(38)$ & $10 / 13(77)$ \\
At CR after 1st line chemotherapy & Group I & 2 & $5 / 6(83)$ & \\
\hline
\end{tabular}

$\mathrm{PD}$, progressive disease; $\mathrm{SD}$, stable disease; $\mathrm{CR}$, complete remission; treat-off, no chemotherapy.

Table III. Comparison of accuracy of cystatin-C ELISA based on tumor status.

\begin{tabular}{lrcc}
\hline & \multicolumn{3}{c}{ Cystatin-C expression, n (\%) } \\
\cline { 2 - 4 } Tumor status & Positive & Negative & Total, n (\%) \\
\hline Present & $22(31.7)$ & $19(30.2)$ & $41(65.1)$ \\
Absent & $8(12.7)$ & $14(22.2)$ & $22(34.9)$ \\
Total & $30(47.6)$ & $33(52.4)$ & $63(100.0)$ \\
\hline
\end{tabular}

rate of cystatin- $\mathrm{C}$ from the tumor mass into the blood is quite low (Table V).
Comparison of serum positivity based on the clinical status of cancer. The blood cystatin-C levels were then compared with the number of metastases from immediate post-operative samples (no residual tumor), up to 5 tumor metastases. The cystatin-C levels were lowest following surgery and increased as the number of metastases increased (Fig. 4A). In 6 patients, the blood cystatin-C levels from both pre- and post-operative samples were compared. Whilst no patients showed positivity for cystatin- $\mathrm{C}$ expression, the expression levels exhibited a reducing trend following surgery $(\mathrm{P}=0.13$; Fig. 4B).

Comparison of serum positivity among cystatin- $C, C A 15-3$ and CA125 in breast cancer. In 92 samples with different states of systemic metastasis, we simultaneously compared 
Table IV. Comparison of cystatin-C expression by immunohistochemistry at diagnosis (pre-chemotherapy) and following surgery (post-chemotherapy).

\begin{tabular}{lll}
\hline \multirow{2}{*}{$\begin{array}{l}\text { Patient } \\
\text { number }\end{array}$} & $\begin{array}{c}\text { Cystatin-C expression } \\
\text { (at diagnosis) }\end{array}$ & $\begin{array}{c}\text { Post-chemotherapy } \\
\text { (following surgery) }\end{array}$ \\
\hline 1 & Positive & Positive \\
2 & Negative & Negative \\
3 & Negative & Positive \\
4 & Negative & Negative \\
5 & Negative & Negative \\
6 & Negative & Positive \\
\hline
\end{tabular}

Table V. Comparison of cystatin-C expression between paired tissue (immunohistochemistry) and blood (ELISA).

\section{Cystatin-C expression on} immunohistochemistry/ELISA

Patients, $\mathrm{n}$

Positive/positive

Positive/negative

Negative/positive

Negative/negative

3

8

2

3

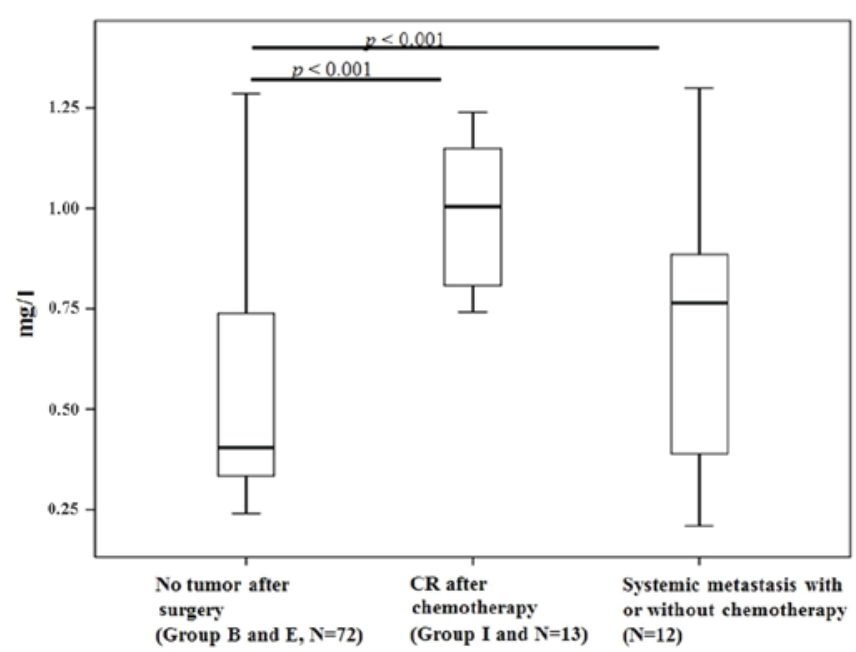

Figure 3. Comparison between cystatin-C levels and tumor burden at different time points. CR, complete remission.

the cystatin-C, CA15-3 and CA125 levels with the numbers of tumor metastases. As the number of metastasis increased, CA15-3 $(\mathrm{P}<0.001), \mathrm{CA} 125(\mathrm{P}=0.001)$ and cystatin- $\mathrm{C}(\mathrm{P}=0.035)$ showed dynamic alterations compared to post-operative (no tumor) state (Fig. 5). The accuracy rates of cystatin-C, CA15-3 and CA125 were 50.0, 67.4 and 59.8\%, respectively. With tumor presence, the concordance rate between cystatin-C and CA15-3, and cystatin-C and CA125 were 66.7 and 53.6\%, respectively. Without tumor presence, the concordance rates
Table VI. Comparison of serum positivity among cystatin-C, CA15-3 and CA125.

A, Tumor presence

\begin{tabular}{lr}
\hline Expression & Total \\
\hline Total & 69 \\
Cystatin-C (+) & 33 \\
Cystatin-C (-) & 59 \\
CA15-3 (+) & 41 \\
CA15-3 (-) & 28 \\
CA125 (+) & 36 \\
CA125 (-) & 33 \\
Cystatin-C (+)/CA15-3 (+) & 23 \\
Cystatin-C (-)/CA15-3 (+) & 18 \\
Cystatin-C (+)/CA15-3 (-) & 23 \\
Cystatin-C (-)/CA15-3 (-) & 16 \\
Cystatin-C (+)/CA125 (+) & 20 \\
Cystatin-C (-)/CA125 (+) & 12 \\
Cystatin-C (+)/CA125 (-) & 21 \\
Cystatin-C (-)/CA125 (-)
\end{tabular}

B, Tumor absence

\begin{tabular}{lr} 
Expression & Total \\
\hline Total & 23 \\
Cystatin-C (+) & 5 \\
Cystatin-C (-) & 18 \\
CA15-3 (+) & 2 \\
CA15-3 (-) & 21 \\
CA125 (+) & 4 \\
CA125 (-) & 19 \\
Cystatin-C (+)/CA15-3 (+) & 2 \\
Cystatin-C (-)/CA15-3 (+) & 0 \\
Cystatin-C (+)/CA15-3 (-) & 3 \\
Cystatin-C (-)/CA15-3 (-) & 18 \\
Cystatin-C (+)/CA125 (+) & 1 \\
Cystatin-C (-)/CA125 (+) & 3 \\
Cystatin-C (+)/CA125 (-) & 4 \\
Cystatin-C (-)/CA125 (-) & 15 \\
\hline CA, cancer antigen.
\end{tabular}

between cystatin-C and CA15-3, and cystatin-C and CA125 were 87.0 and $69.6 \%$, respectively (Table VI).

\section{Discussion}

Cystatin-C is a non-glycosylated low molecular weight basic protein and is the product of a housekeeping gene, CST-3 (19). Cystatin- $\mathrm{C}$ is constitutively produced by all human nucleated cells. Blood cystatin-C levels are independent of age, body mass index (20) and sex (15) in healthy individuals (20); however, the levels show a weak association with age due to 
A

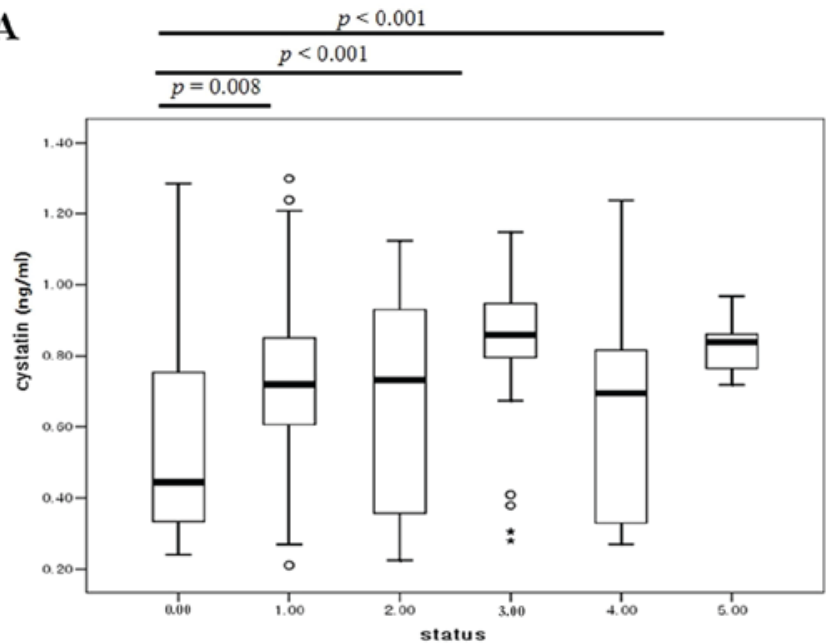

B

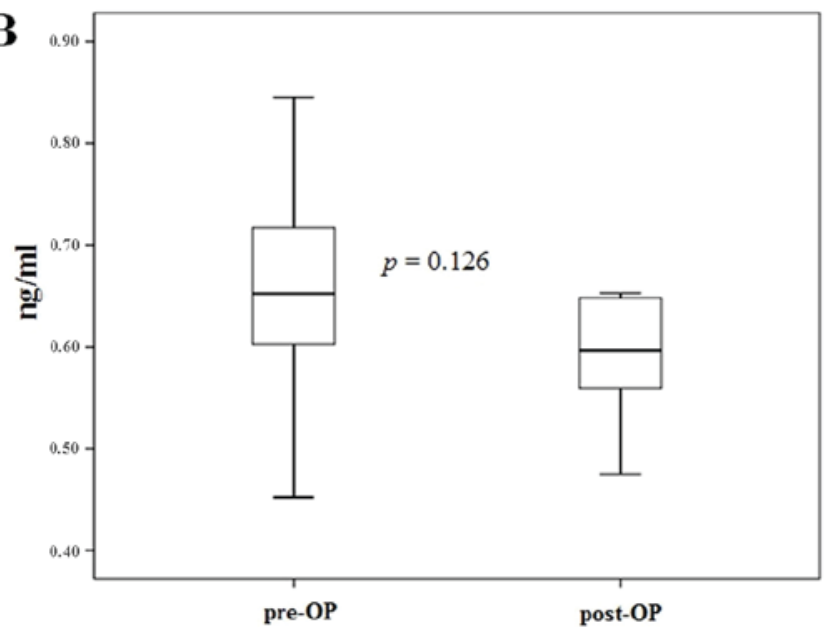

Figure 4. Comparison of cystatin-C levels (A) based on the number of tumor metastasis organs and (B) between pre-op and post-op states of primary breast cancer. Pre-op, pre-operative; post-op, post-operative.

a reduced glomerular filtration rate in elderly individuals (21). Finney et al (20) reported no difference in cystatin-C levels by age, but the median age of the control group in their study was 40 years (range, 19-59 years), while Norlund et al (21) reported an age dependency in older individuals. The present study tested blood cystatin-C levels in healthy Korean volunteers (age range, 21-56 years), which indicated that there was no age-dependence in males or females. However, the levels were increased in males compared with in females, potentially due to a difference in age. The median age of the men was 41 years (range, 25-66 years) and in the women was 35 years (range, 21-56 years). Ohara et al (16) reported age dependence in cystatin-C levels in elderly patients with lung cancer aged $>75$ years old compared with those aged $<65$ years old (16). In patients with colon cancer, the cystatin-C levels were 1.4-fold increased in all stages of cancer compared with healthy individuals (15). In breast cancer, inflammatory cancer type and increased tumor size exhibited high expression levels of cystatin-C (17). Although the control group was not age-matched with the patient group in the present study, patients with breast cancer exhibited increased levels of cystatin-C
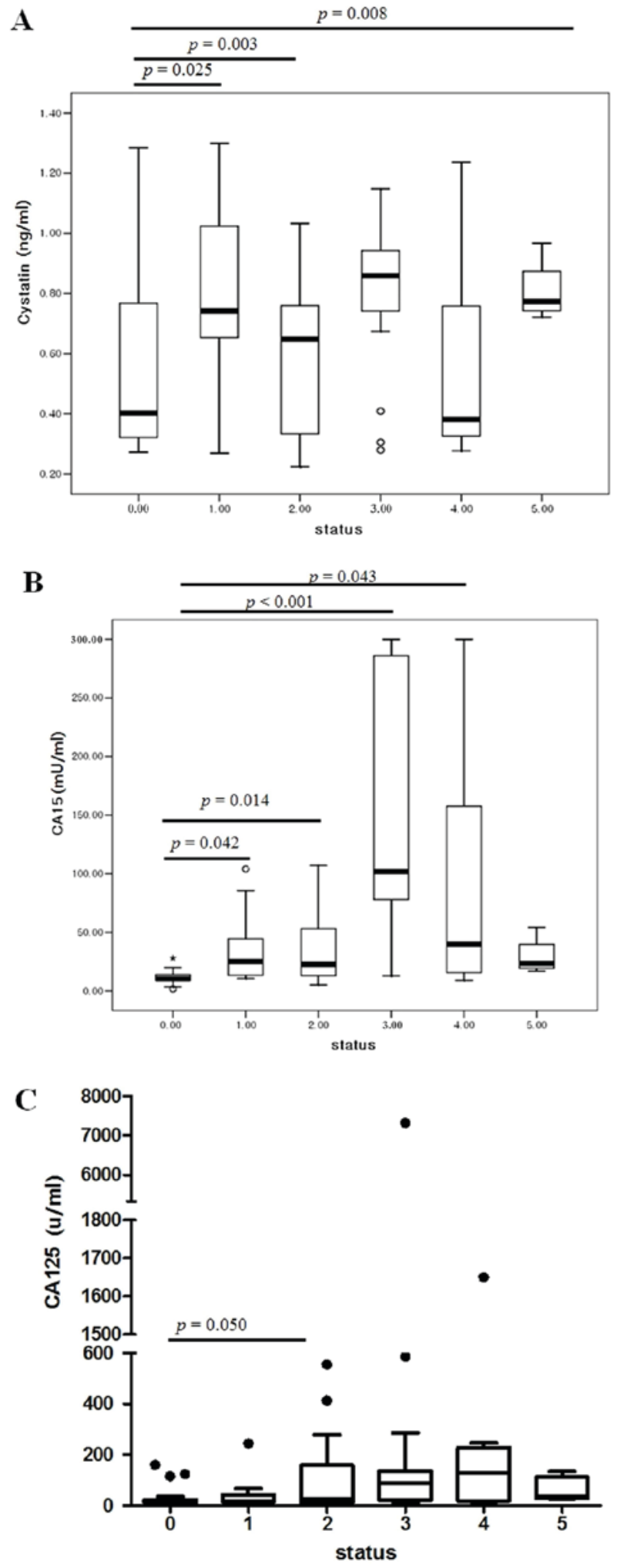

Figure 5. Comparison of (A) cystatin-C, (B) CA15-3 and (C) CA125 levels based on the number of tumor metastasis organs. CA, cancer antigen.

compared with healthy volunteers. Due to the availability of 205 blood samples from 34 patients with breast cancer at 12 different clinical time points, the cystatin-C positive rate was evaluated at the different time points. This demonstrated the tumor mass dependence of cystatin-C level, by the observation of the lowest serum positivity following surgery, where 
there is no tumor. However, despite the increased cystatin-C levels in patients with cancer, the sensitivity of the blood test was only $53.6 \%$ and the accuracy was $53.9 \%$, suggesting a low secretion capacity from the tumor mass.

Due to the low blood positivity rate, immunohistochemical analysis of cystatin- $\mathrm{C}$ was conducted to investigate the expression levels of cystatin- $\mathrm{C}$ in primary breast cancer. The expression levels were evaluated at 3 different time points (at diagnosis, following pre-operative chemotherapy and at relapse), resulting in a positivity rate of $54.0 \%$, which was similar to the blood positivity. The positivity rate increased from diagnosis to relapse, suggesting an association between tumor progression and cystatin-C expression. In addition, synchronicity was investigated in 6 paired samples by comparing samples taken at diagnosis, before chemotherapy, with samples taken following chemotherapy and surgery, which resulted in a concordance rate of $66.7 \%$. Two initially negative patients exhibited cystatin- $\mathrm{C}$ expression following chemotherapy. This may be due to tumor heterogeneity from the small biopsy specimen or cystatin- $\mathrm{C}$ induction subsequent to chemotherapy. In previous studies, no differences in cystatin- $\mathrm{C}$ levels were observed in samples from prior to and subsequent to chemotherapy (median age, 57 years) (22), while transient increments (20-40\%) were detected following chemotherapy (within 10 days of chemotherapy) in esophageal cancer (median age, 60 years) (23). This requires further confirmation through the investigation of an increased number of cases.

As the positivity rate was similar in blood and tissue levels, tissue expression and blood levels were then compared in 16 patients. The concordance rate was only $38 \%$, which mostly resulted from the low blood positivity $(27 \%)$ in the tissue positive cases. This suggests that the blood cystatin-C levels are associated with tumor volume; however, secreted cystatin-C levels in the blood are quite low in early breast cancer. Laurent-Matha et al (24) reported the amount of cystatin-C in the extracellular environment is reduced when the cell is transfected with cathepsin D. In addition, cathepsin D secreted by breast cancer cells extensively cleaved cystatin-C, which may reduce the cystatin-C levels in the blood (24).

To investigate the association between tumor volume and blood cystatin-C levels, the cystatin-C levels were compared with the number of metastases. Cystatin- $C$ was observed to be at the lowest levels subsequent to surgery, when the tumor had been removed, with the levels increasing as the number of metastases increases. As in the immunohistochemical analysis, paired comparisons were performed between cystatin-C levels prior and subsequent to surgery in 6 patients. Whilst these patients did not show serum positivity, the absolute blood levels of cystatin-C decreased subsequent to surgery compared with the time of diagnosis, suggesting levels are dependent upon the tumor volume. Similarly in ovarian cancer, cystatin-C has been reported to increase only in patients with cancer, not in healthy individuals and patients with benign ovarian disease (25). By contrast, cystatin-C levels were low in prostate cancer specimens compared with benign tissues. Wegiel et al (26) observed an inverse correlation between the expression of cystatin-C and matrix metalloproteinase 2. Similarly, the present study did not find any patients with prostate cancer to be cystatin-C positive, suggesting a functional specificity of cystatin- $\mathrm{C}$ in different types of cancer, due to the presence of different co-functioning molecules. Cystatin- $\mathrm{C}$ is a TGF- $\beta$ receptor antagonist in addition to a cystatin-C-mediated feedback loop that inhibits TGF- $\beta$ signaling (27). By inhibiting oncogenic TGF- $\beta$ signaling, cystatin- $C$ is effective in preventing breast cancer angiogenesis and progression (28).

As a cysteine protease inhibitor, it was reported that cystatin- $C$ reduces cancer invasion and metastasis (25). Cystatin- $\mathrm{C}$ was increased in cancer tissues from ovarian cancer, non-small cell lung cancer and in blood samples from patients with hepatocellular carcinoma, melanoma, breast cancer and colon cancer. By contrast, cystatin-C mRNA levels did not change in pancreatic, head and neck cancer. Using laser-capture microdissection technology, breast cancer cells were reported to show increased expression levels of cystatin- $\mathrm{C}$, with this correlating with increased tumor size (29). However, the increased cystatin-C expression relative to cathepsin B (9) and cathepsin D (24) expression was reduced. Cathepsin $\mathrm{X}$ and $\mathrm{H}$ were significantly lower in inflammatory breast cancer compared with cystatin- $C$ levels (17). This imbalance between cysteine protease and proteinase inhibitors may be associated with the ability of cancer interstitial tissues to detach, which is important in cancer invasion and metastasis (9). High levels of stefin B and cystatin- $\mathrm{C}$ were associated with poor prognosis, whether as a result of primary activation or secondary feedback loop activation (15). To investigate whether the elevated levels of cystatin- $\mathrm{C}$ were associated with tumor mass, we then compared dynamic alterations in cystatin- $\mathrm{C}$ levels based on tumor volume with known tumor markers in breast cancer, such as CA15-3 and CA125. Cystatin-C showed a plateau in the blood levels as the tumor mass increased, while CA15-3 showed continuous increases in the blood level with tumor mass increases. The concordance rates of cystatin-C and CA15-3, cystatin-C and CA125 were low (66.7 and 53.6\%) when tumors were present compared with when tumors were absent (87.0 and 69.6\%). This indicates that a minimum of one-third of breast cancer showed molecular tumor heterogeneity in the expression of cystatin-C and CA15-3.

In conclusion, elevated blood cystatin- $\mathrm{C}$ levels were detected in $40 \%$ of breast cancer cases, with this being tumor volume-dependent. However, the concordance rate between levels in tissue and blood samples was low, suggesting tumor heterogeneity in cystatin-C expression or co-acting pathway activation, such as cathepsin D. As one-third of breast cancer cases expressed cystatin-C without CA15-3 elevation, cystatin- $\mathrm{C}$ may represent a good tumor-monitoring marker in breast cancer.

\section{References}

1. Lankisch P, Wessalowski R, Maisonneuve P, Haghgu M, Hermsen D and Kramm CM: Serum cystatic C is a suitable marker for routine monitoring of renal function in pediatric cancer patients, especially of very young age. Pediatr Blood Cancer 46: 767-772, 2006.

2. Chew-Harris JS, Florkowski CM, George PM and Endre ZH: Comparative performances of the new chronic kidney disease epidemiology equations incorporating cystatin- $\mathrm{C}$ for use in cancer patients. Asia Pac J Clin Oncol 11: 142-151, 2015.

3. Magnusson M, Hedblad B, Engström G, Persson M, Nilsson P and Melander O: High and Cancer Study. J Int Med 274: 192-199, 2013. 
4. Sheahan K, Shuja S and Murnane MJ: Cysteine protease activities and tumor development in human colorectal carcinoma. Cancer Res 49: 3809-3814, 1989.

5. Hirai K, Yokoyama M, Asano G and Tanaka S: Expression of cathepsin B and cystatin-C in human colorectal cancer. Human Pathol 30: 680-686, 1999.

6. Saleh Y, Sebzda T, Warwas M, Kopec W, Ziólkowska J and Siewinski M: Expression of cystatin c in human colorectal cancer tissues. J Exp Ther Oncol 5: 49-53, 2005.

7. Zeng Q, Zhao Y, Yang Y, Zheng G, Wang G, Zhang P, Cui Y, $\mathrm{Su} \mathrm{S}$ and Li K: Expression of cystatin $\mathrm{C}$ in human esophageal cancer. Tumori 97: 203-210, 2011.

8. Jiborn T, Abrahamson M, Gadaleanu V, Lundwall A and Bjartell A: Aberrant expression of cystatin C in prostate cancer is associated with neuroendocrine differentiation. BJU Int 98: 186-196, 2006

9. Yano M, Hirai K, Naito Z, Yokoyama M, Ishiwata T, Shiraki Y, Inokuchi $\mathrm{M}$ and Asano $\mathrm{G}$ : Expression of cathepsin $\mathrm{B}$ and cystatin C in human breast cancer. Surg Today 31: 385-389, 2001.

10. Sloane BF: Cathepsin B and cystatins: Evidence for a role in cancer progression. Cancer Biol 1: 137-152, 1990.

11. Utsunomiya T, Hara Y, Kataoka A, Morita M, Arakawa H, Mori M and Nishiyama S: Cystatin-like metastasis-associated protein mRNA expression in human colorectal cancer is associated with both liver metastasis and patient survival. Clin Cancer Res 8: 2591-2594, 2002.

12. Kuopio T, Kankaanranta A, Jalava P, Kronqvist P, Kotkansalo T, Weber E and Collan Y: Cysteine proteinase inhibitor cystatin A in breast cancer. Cancer Res 58: 432-436, 1998.

13. Leto G, Tuminello FM, Pizzolanti G, Montallo G, Soresi M and Gebbia N: Lysosomal cathepsins B and L and stefin A blood levels in patients with hepatocellular carcinoma and/or liver cirrhosis: Potential clinical implications. Oncology 54: 79-83, 1997.

14. Kos J, Stabuc B, Schweiger A, Krasovec M, Cimerman N, Kopitar-Jerala N and Vrhovec I: Cathepsin B, H, L and their inhibitors stefin $\mathrm{A}$ and cystatin- $\mathrm{C}$ in sera of melanoma patients. Clin Cancer Res 3: 1815-1822, 1997.

15. Kos J, Krasovec M, Cimerman N, Nielsen HJ, Christensen IJ and Brunner N: Custeine proteinase inhibitors stefin-A, stefin-B, and cystatin-C in sera from patients with colorectal cancer: Relation to prognosis. Clin Cancer Res 6: 505-511, 2000.

16. Ohara G, Miyazaki K, Kurishima K, Kagohashi K, Ishikawa H, Satoh $\mathrm{H}$ and Hizawa N: Serum levels of cystatin-C in elderly lung cancer patients. Oncol Lett 3: 303-306, 2012.

17. Decock J, Obermajer N, Vozelj S, Hendricks W, Paridaens R and Kos J: Cathepsin B, cathepsin H, cathepsin X and cystatin C in sera of patients with early-stage and inflammatory breast cancer. Int J Biol Markers 23: 161-168, 2008.

18. Rha SY, Yang WI, Gong SJ, Kim JJ, Yoo NC, Roh JK, Min JS Lee KS, Kim BS and Chung HC: Correlation of tissue and blood plasminogen activation system in breast cancer. Cancer Lett 150 $137-145,2000$
19. Hwang SJ, Yang Q, Meigs JB, Pearce EN and Fox CS: A genome-wide association for kidney function and endocrine-related traits in the NHLBI's Framingham Heart Study. BMC Med Genet 8 (Suppl 1): S10, 2007.

20. Finney H, Bates CJ and Price CP: Plasma cystatin C determinations in healthy elderly population. Arch Gerontol Geriatr 29: 75-94, 1999.

21. Norlund L, Grubb A, Fex G, Leksell H, Nilsson JE, Schenck H and Hultberg B: The increase of plasma homocysteine concentrations with age is partly due to the deterioration of renal $\mathrm{f}$ unction as determined by plasma cystatin C. Clin Chem Lab Med 36: 175-178, 1998.

22. Stabuc B, Vrhovec L, Stabuc-Silih M and Cizej TE: Improved prediction of decreased creatinine clearance by serum cystatin C: Use in cancer patients before and during chemotherapy. Clin Chemis 46: 193-197, 2000.

23. Kume M, Yasui H, Yoshikawa Y, Horinouchi M, Higashiguchi K, Kobayashi Y, Kuroda D, Hirano T, Hirai M and Nakamura T: Transient elevation of serum cystatin $\mathrm{C}$ concentrations during perioperative cisplatin-based chemotherapy in esophageal cancer patients. Cancer Chemother Pharmacol 69: 1537-1544, 2012.

24. Laurent-Matha V, Huesgen PF, Masson O, Derocq D, Prébois C Gary-Bobo M, Lecaille F, Rebiére B, Meurice G, Oréar C, et al: Proteolysis of cystatin- $\mathrm{C}$ by cathepsin $\mathrm{D}$ in the breast cancer microenvironment. FASEB J 26: 5172-5181, 2012.

25. Nishikawa H, Ozaki Y, Nakanishi T, Blomgren K, Tada T, Arakawa A and Suzumori K: The role of cathepsin B and cystatin $\mathrm{C}$ in the mechanisms of invasion by ovarian cancer. Gynecol Oncol 92: 881-886, 2004

26. Wegiel B, Jiborn T, Abrahamson M, Helczynski L, Otterbein L, Persson JL and Bjartell A: Cystatin C is downregulated in prostate cancer and modulates invasion of prostate cancer cells vis MAPK/Erk and androgen receptor pathways. PLos One 4: e7953, 2009.

27. Sokol JP and Schiemann WP: Cystatin C antagonizes transforming growth factor beta signaling in normal and cancer cells. Mol Cancer Res 2: 183-195, 2004.

28. Tian M and Schiemann WP: Preclinical efficacy of cystatin C to target the oncogenic activity of transforming growth factor Beta in breast cancer. Transl Oncol 2: 174-183, 2009.

29. Vigneswaran N, Wu J, Muller S, Zacharias W, Narendran S and Middleton L: Expression analysis of cystatin $\mathrm{C}$ and $\mathrm{M}$ in laser-capture microdissectioned human breast cancer cells-a preliminary study. Pathol Res Pract 200: 753-762, 2005.

This work is licensed under a Creative Commons Attribution-NonCommercial-NoDerivatives 4.0 International (CC BY-NC-ND 4.0) License. 